\title{
Measurement of Soil Water Potential
}

\author{
Gary A. Clark ${ }^{1}$ \\ Gulf Coast Research and Education Center, University of Florida, Bradenton, Florida 34203
}

Total soil water potential includes pressure, gravimetric, and osmotic components. Measurement of gravimetric potential is relatively easy because it is referenced to a fixed datum. Unsaturated pressure potentials and osmotic potentials are more difficult to measure. Generally, another property of the soil is measured and calibrated to the water potential, or a reference matrix is used to equilibrate with the soil and then a property of that reference matrix is measured. Common devices used for measuring soil water potential include tensiometers, electrical resistance blocks, heat pulse (or

Fla. Agr. Expt. Sta. J. Ser. no. R-01071.

${ }^{1}$ Assistant Professor of Agricultural Engineering. thermal conductivity) sensors, and thermocouple psychrometers.

Soil water potential represents the static energy state of soil water and its potential energy. Movement of water within the soil is slow; thus, the other form of energy, kinetic, is considered to be negligible. Soil water potential is considered to be the "total" energy level with respect to the energy state of soil water and is generally expressed in terms relative to water in a standard reference state.

\section{TOTAL SOIL WATER POTENTIAL}

Several potential energy components combine to form the total soil water potential. These energy components result from the force fields generated by the adsorption of water to solid surfaces (soil 
particles), the presence of solutes, external air or gas pressures, and gravitational forces (Campbell, 1987; Hillel, 1980). The contributions of the different components are summed in the expression:

$$
\Phi_{\mathrm{t}}=\Phi_{\mathrm{p}}+\Phi_{\mathrm{s}}+\Phi_{\infty}
$$

where $\phi_{1}$ is the total potential, $\phi_{\mathrm{p}}$ is the water pressure potential, $\phi_{\mathrm{g}}$ is the gravitational potential, and $\phi_{\mathrm{o}}$ is the osmotic potential.

Additional terms could be defined and included, but would not have the significance of the defined terms and are left out for convenience. Pneumatic potential (air pressure) is added to the total potential when differences from atmospheric pressure exist. However, this discussion deals with normal atmospheric conditions only and uses pressure potential to imply water pressure potential.

\section{Pressure potential}

Soil conditions of saturation or unsaturation affect the sign $(+/-)$ of the pressure potential term with respect to the reference state. When soil is saturated, the soil water is at a hydrostatic pressure greater than atmospheric and is positive with respect to the reference state. In unsaturated conditions, the capillary and adsorptive forces of the soil matrix to the water create a negative pressure potential.

Sometimes the terms matric potential, matric suction, or tension (all positive terms) are used interchangeably, which can create confusion with respect to pressure potential. Pressure potentials will always be negative in unsaturated soils. If pressure potentials are strictly adhered to, there should never be any confusion with regard to the sign $(+/-)$.

Pressure potential is positive under saturated conditions. The hydrostatic pressure $\mathrm{P}$ of the water at a point with respect to atmospheric pressure is

$$
P=\Gamma h,
$$

where $\Gamma$ is the specific weight of water and $h$ is the piezometric head.

In unsaturated soils, when the presence of solutes is negligible or nonexistent, the pressure potential of water in the vapor phase can be calculated from the relative humidity. The humidity in the vapor phase and water potential of the soil are related at equilibrium by

$$
\Phi_{\mathrm{p}}=\mathrm{RT} \ln \left(\mathrm{p} / \mathrm{p}^{\prime}\right)
$$

where $\mathrm{R}$ is the gas constant for water vapor, $\mathrm{T}$ is the absolute temperature (degrees $\mathrm{K}$ ), and $\mathrm{p} / \mathrm{p}^{\prime}$ is the relative humidity (the ratio of the partial pressure of vapor in the air phase to the partial pressure at saturation). When solutes are present, this relationship yields the water potential consisting of both the pressure and osmotic components.

\section{Gravitational potential}

The gravitational potential depends only on relative elevation with respect to a fixed reference. The soil surface is commonly used as the reference datum resulting in negative gravitational potentials for all points below the surface. The gravitational potential energy can be determined from

$$
\Phi_{\mathrm{g}}=\Gamma Z,
$$

where $\mathrm{z}$ is the elevation of the point in question with respect to the reference elevation.

\section{Osmotic potential}

The osmotic potential influences water flow only when a gradient in solute concentration exists across a semipermeable membrane or diffusion barrier. The osmotic potential is proportional to the concentration of the solute particles and the solution temperature and can be determined from modifying Eq. [3]:

$$
\pi=\text { MRT }
$$

where $\pi$ is the osmotic potential, $\mathrm{M}$ is the molar concentration of solute particles, $\mathrm{R}$ is the gas constant, and $\mathrm{T}$ is the absolute temperature (degrees $\mathrm{K}$ ).

\section{MEASUREMENT OF SOIL WATER POTENTIAL}

Total soil water potential was described by Eq. [1]. The sum of pressure and osmotic potentials is sometimes referred to as water potential. When pressure potential is combined with gravitational potential, the sum is referred to as the hydraulic potential. The hydrostatic pressure and gravitational potential were described in Eqs. [2] and [4], respectively. Measurement of these properties is relatively easy. Both can be measured with a ruler or linear measurement scale. A piezometer may be necessary to determine the level of the free water surface for hydrostatic pressure measurements.

\section{Pressure potential}

Several direct and indirect measurement techniques are available for measuring pressure potential (Campbell, 1987). Instruments placed in the soil matrix and allowed to attain hydraulic equilibrium could provide a direct measure of pressure potential. Tensiometers are the most common instrument used for this purpose. Resistance blocks and heat pulse or thermal conductivity blocks equilibrate with the pressure potential of the soil, but do not provide a direct indication of pressure potential. The measured property is calibrated against the pressure potential/water content relationship of the porous medium used in the device.

Measuring the water content of the soil matrix to determine the corresponding pressure potential by using the relationship between pressure potential and water content for that soil provides an indirect estimate of pressure potential (Hillel, 1980). Water content can be measured or estimated by several techniques such as gravimetric sampling, radiation methods (neutron, gamma, microwave, ....), time domain reflectometry (TDR), and others.

Tensiometers. The porous tensiometer cup is generally made of ceramic and is permeable to water and solutes. Air will pass through the cups at varying levels of negative pressure depending on the quality and make of the porous cup material. The negative pressure sensing device may be a vacuum gauge, manometer, or pressure transducer.

The pressure potential of the porous cup equilibrates with the pressure potential of the surrounding soil medium. Osmotic potentials do not have an effect on the tensiometer reading. The porous cup must have continuous contact with the surrounding soil to ensure an accurate reading of the soil pressure potential. Air gaps will result in erroneous measurements.

The negative pressure sensors vary in accuracy as well as cost. Vacuum gauges are perhaps the easiest device to use and are very suitable for multiple sites in field use. Accuracy of most gauges is limited to the sensitivity of the gauge, which is generally $2 \mathrm{kPa}$. When using gauges, it is recommended that all of the air be extracted. Hand-operated vacuum pumps may be used for this purpose. These gauges contain spring- or diaphragm-operated parts and may require periodic calibration. Some gauges permit adjustment of the needle for calibration.

Water or mercury manometers may be used if greater measurement sensitivity is required. Mercury manometers can provide accuracies of $0.2 \mathrm{kPa}$, although $0.5 \mathrm{kPa}$ is more realistic. A disadvantage of this method of measurement is that several sites must be located within close proximity to take advantage of each manometer station. Care in handling mercury is recommended due to the toxicity of the vapors and the corrosive action mercury has for some other metals.

Pressure transducers will also provide good measurement sensitivity $(0.1$ to $0.2 \mathrm{kPa})$. Cost may limit the use of these devices. Generally, one pressure transducer is wired to a data recorder and then connected to each tensiometer for measurement. Manual operations allow multiple field sites to be monitored with a minimum of hydraulic connections. Automation may be cumbersome in the field, but these instruments are well-suited for laboratory or field installations where instruments and data loggers are in close proximity. 
The useful limit of a tensiometer is about $-85 \mathrm{kPa}$. Limitations result from the failure of water columns in the manometers at or near pressure potentials of $-100 \mathrm{kPa}$, and entry of air into the porous cups equalizing internal pressures of the tensiometer to atmospheric. This is not a problem for most applications. The soil pressure potential range of 0 to $-85 \mathrm{kPa}$ encompasses the major portion of the soil wetness range acceptable for plant growth.

Electrical resistance blocks. The electrical resistance of a porous medium depends on water content, soluble salt content, structure, and texture. Standardized blocks of porous media are used eliminate variations due to structure, texture, and, to some extent. salinity.

Commercial electrical resistance blocks are constructed of gypsum, nylon, fiberglass, or some other reference matrix material (Armstrong et al., 1987; Carlson and El Salem, 1987). A pair of electrodes used to measure electrical resistance (or conductance) is embedded in the reference matrix block. Thus, electrical resistance is usually calibrated against the water content of the block. It is important to remember these blocks must equilibrate with the pressure potential of the surrounding soil and additional calibrations will be required to obtain the pressure potential and/or water content of the soil medium.

Salinity causes problems with calibration. Blocks made of materials such as nylon or fiberglass provide no electrolytic buffer and are highly sensitive to changes in salinity of the soil solution. Gypsum blocks maintain a saturated solution of calcium sulfate that tends to mask salinity changes in the soil solution. Gypsum blocks dissolve under wet conditions and require periodic replacement.

Thermal conductivity. Thermal conductivity of a porous medium depends on the mineral content, structure, and moisture content (Barnes et al., 1981). If the textural, structural, and mineral components can be standardized, water content will be the primary influence on thermal conductivity. Commercially available blocks of ceramic are used to determine the pressure potential of the soil matrix by a heat pulse method (Phene et al., 1987). Ceramic blocks provide a standard matrix such that the primary influence on the thermal properties is water content and hence pressure potential. The devices can be calibrated against water content or pressure potential. Similar to the electrical resistance blocks in use (but not in theory of operation), these devices are embedded in the soil medium and allowed to reach hydraulic equilibrium. Due to the nature of these devices, soil structure and salinity should not influence measurements (Phene et al., 1987).

\section{Osmotic potential}

Measurement of osmotic potential alone is more difficult than measuring pressure potential. Perhaps the most reliable measurement technique involves extracting a soil solution, then through measurement of the solute concentration the osmotic potential can be calculated (Eq. [5]). Another method would involve measuring the combined pressure and osmotic potential (water potential) and extrapolating the latter through an independent measure of pressure potential.

\section{Pressure and osmotic potential-water potential}

Equation 3 was presented as a means of calculating the pressure potential of a porous matrix when solute levels are negligible. That same relationship represents the water potential of a porous medium when the solute levels are not negligible and is the basis for psychrometric measurements.

The use of psychrometry to measure soil water potential has been discussed and evaluated by several researchers (Campbell et al., 1972; Dalton and Rawlins, 1968; Klute and Richards, 1962; Merrill and Rawlins, 1972; Rawlins and Dalton, 1967). Thermocouple psychrometers are commercially available devices that are used to measure the temperature and relative humidity of a moist air sample and thus provide the necessary data to calculate water potential (Briscoe, 1984; Wescor, 1986).

Soil psychrometers are used to measure the water potential of the soil atmosphere with a range of 0 to $-5000 \mathrm{kPa}$ (Brown and Chambers, 1987). The measurement is accomplished by placing one thermocouple junction inside a miniature porous cup 2 nd another junction in an insulated medium. The device is buried in the soil and allowed to equilibrate with the hydraulic potential of the soil. The atmosphere inside the porous cup equilibrates with the water potential of the porous cup matrix. Then the thermocouple junction in the porous cup is used to measure the relative humidity of that atmosphere while the other thermocouple junction is used to measure ambient soil temperature. Basic methods to measure humidity within the porous cup are the psychrometric or wet bulb and the hygrometric or dew point (Wescor, 1986). Both psychrometric and hygrometric methods require a microvoltmeter to measure the thermocouple output, a strip chart recorder to monitor the thermocouple output and rates of change, and a temperature-controlled water bath or ice-point compensator.

Humidity is measured in the psychrometric method by determining wet bulb depression (Briscoe, 1984). The wet bulb temperature is determined by observing the thermocouple output on the strip chart recorder. The thermocouple in the porous cup is cooled below the dew point by means of the Peltier effect. The Peltier current is discontinued when a film of water has formed on the junction surface. The temperature of the thermocouple coated with water will increase steadily until the wet bulb temperature is reached. Subsequent evaporation of the water cools the junction and offsets the heat absorbed from the surroundings which, in turn, reduces the rate of change in the thermocouple output until the water has evaporated. After the water has evaporated, the thermocouple returns to ambient temperature with a greater rate of change in the thermocouple output. The period of evaporative cooling is somewhat transitory and therefore introduces the potential for errors in assessing true wet bulb temperature.

Dew point temperature is measured and used to calculate humidity in the hygrometric method. The thermocouple in the porous cup is again cooled below the dew point as in the psychrometric method. Temperature of the thermocouple junction is then controlled by offsetting the heat gains and losses by Peltier cooling. The thermocouple temperature converges to the dew point where it will remain with a static amount of water. This procedure tends to maintain a more stable condition, thus a longer period for measurement and greater precision than the wet bulb method.

These are temperature-dependent methods, thus temperature fluetuations and gradients can result in significant errors. The effects of thermal gradients on in situ measurements of soil water potential have been discussed by several researchers (Brown and Chambers, 1987; Campbell and Gardner, 1971; Dalton and Rawlins, 1968; Hsieh and Hungate, 1970; Merrill and Rawlins, 1972; Rawlins and Dalton, 1967; Wiebe and Brown, 1979; Wiebe et al., 1977). Calibration with accurate temperature control and monitoring is necessary. A temperature gradient within the psychrometer before Peltier cooling will produce a microvoltage output called a null voltage or "zero offset" (Weibe et al., 1977). A temperature correction must be applied to the calibration measurements as well as to field measurements (Briscoe, 1984). Soil psychrometers/hygrometers can be difficult to calibrate because they do not have a large heat sink attached to the thermocouple. A $0.5-\mathrm{m}$ length of lead wire in the calibration container can prevent heat flux from travelling along the lead wire to the thermocouple. A water bath can be used to control the temperature of the sensors during calibration and to minimize thermal gradients. The soil psychrometers/hygrometers should be installed with the axis of the sensor parallel to the soil surface. This geometry provides an orientation perpendicular to the direction of radiant heat flux and can reduce the total thermal gradient across the sensor.

\section{Literature Cited}

Armstrong, C.F., J.T. Ligon, and M.F. McLeod. 1987. Automated system for dctailed measurement of soil water potential profiles using watermark brand sensors. Proc. Intl. Conf. Measurement Soil Plant Water Status. Logan, Utah 1:201-206.

Barnes. P.L., A.G. Smajstrla, and D.L. Reddell. 1981. Thermal conductivity related to moisture content in soils. 1981 Amer. Soc. Agr. Eng. Winter Meeting. Chicano. ASAE Paper 81-2511.

Briscoe. R.D. 1984. Thermocouple psychrometers for water potential measurements. Proc. NATO Advanced Study Inst. on Advanced Agr. Instrumentation. II Ciocco (Pisa). Italy. 
Brown, R.W. and J.C. Chambers. 1987. Measurements of in situ water potential with thermocouple psychrometers: a critical evaluation. Proc. Intl. Conf. Measurement Soil Plant Water Status, Logan, Utah. 1:125135.

Campbell, G.S. 1987. Soil water potential measurement. Proc. Intl. Conf. Measurement Soil Plant Water Status, Logan, Utah. 1:115-118.

Campbell, G.S. and W.H. Gardner. 1971. Psychrometric measurement of soil water potential: temperature and bulk density effects. Soil Sci. Soc. Amer. Proc. 35:8-12.

Campbell, E.C., G.S. Campbell, and W.K. Barlow. 1972. A dew point hygrometer for water potential measurement. 1972 Amer. Soc. Agr. Eng. Summer Meeting, Hot Springs, Ark. ASAE Paper 72-436.

Carlson, T.N. and J. El Salem. 1987. Measurement of soil moisture using gypsum blocks. Proc. Intl. Conf. Measurement Soil Plant Water Status, Logan, Utah 1:193-200.

Dalton, F.N. and S.L. Rawlins. 1968. Design criteria for Peltier-effect thermocouple psychrometers. Soil Sci. 105:12-17.

Hillel, D. 1980. Fundamentals of soil physics. Academic, New York.

Hsieh, J.J.C. and F.P. Hungate. 1970. Temperature compensated Peltier psychrometer for measuring plant and soil water potentials. Soil Sci. 110:253-257.

Klute, A. and L.A. Richards. 1962. Effect of temperature on relative vapor pressure of water in soil: apparatus and preliminary measurements. Soil Sci. 93:391-396

Merrill, S.D. and S.L. Rawlins. 1972. Field measurement of soil watcr potential with thermocouple psychrometers. Soil Sci. 113: 102-109.

Phene, C.J., C.P. Allee, and J. Pierro. 1987. Measurement of soil matric potential and real time irrigation scheduling. Proc. Intl. Conf. Measurement Soil Plant Water Status, Logan, Utah 2:258-265.

Rawlins, S.L. and F.N. Dalton. 1967. Psychrometric measurement of soil water potential without precise temperature control. Soil Sci. Soc. Amer. Proc. 31:297-301.

Wescor. 1986. Water potential system. Wescor, Inc. (catalog).

Wiebe, H.H. and R.W. Brown. 1979. Temperature gradient effects on in situ hygrometer measurements of soil water potential. II. water movcment. Agron. J. 71:397-401.

Wiebc, H.H., R.W. Brown, and J. Barker. 1977. Temperature gradient effects on in situ hygrometer measurements of soil water potential. Agron. J. 69:933-939. 